\title{
A Review of Clinical Tests and Signs for the Assessment of Ulnar Neuropathy
}

\author{
Sarah B. Goldman, PhD, OTR/L, CHT \\ Department of Kinesiology, Indiana University, \\ Bloomington, Indiana
}

\section{Teresa L. Brininger, PhD, OTR/L, CHT Military Performance Division, U.S. Army Research Institute of Environmental Medicine, Natick, Massachusetts}

\author{
John W. Schrader, HSD, ATC \\ David M. Koceja, PhD \\ Department of Kinesiology, Indiana University, \\ Bloomington, Indiana
}

Clinical testing is an essential component in identifying and determining the extent of pathology for many upper extremity conditions; however, it can be particularly useful for evaluating patients with ulnar neuropathy. Injury to the ulnar nerve often results in a predictable set of upper extremity sensory and motor impairments. Sensory disturbances typically involve the small and ring fingers and can also involve the dorsal-ulnar aspect of the hand if the lesion is proximal to the wrist. ${ }^{1}$ Sensory changes can contribute to motor control difficulties or even manifest as a safety issue with severe compression to afferent axons transmitting pain and temperature information. Ulnar nerve motor involvement affects most of the intrinsic hand muscles, excluding three muscles in the thenar eminence and the lumbrical muscles to the index and ring fingers. If the entrapment is at the level of the elbow, the flexor digitorum profundus (FDP) to the ring

\footnotetext{
The opinions or assertions contained herein are the private views of the author(s) and are not to be construed as official or as reflecting the views of the Department of the Army or the Department of Defense.

Correspondence and reprint requests to Sarah B. Goldman, PhD, OTR/L, CHT, U.S; Army Research Institute of Environmental Medicine, Kansas Street, Building \#42, Natick, MA 01760; e-mail: <sarah.goldman@us.army.mil>.

0894-1130/\$ - see front matter (C) 2009 Hanley \& Belfus, an imprint of Elsevier Inc. All rights reserved.

doi:10.1016/j.jht.2008.10.010
}

\begin{abstract}
:
Narrative Review:

As part of a comprehensive assessment for suspected ulnar neuropathy, clinical testing plays an important role in the initial identification of a lesion and determining subsequent changes from baseline. The purpose of this article was to review ulnar nerve provocative testing and the substantial collection of diagnostic signs and tests. Administration procedures for each maneuver are described as well as the resulting positive and negative outcomes. The clinical tests described constitute only one aspect of the examination and should not substitute for other key components, such as taking a thorough medical and occupational history. Empirical research studies are indicated to further quantify the relationship between the testing outcomes and the severity of a lesion as well as to determine the most robust motor signs seen in the early stages of the disease.

Level of Evidence: 5.

J HAND THER. 2009;22:209-20.
\end{abstract}

and small fingers and the flexor carpi ulnaris (the strongest wrist flexor) may become weakened. ${ }^{1}$ Other symptoms associated with peripheral motor axon involvement include muscle cramps and fasciculations. ${ }^{2}$ Chronic entrapment of ulnar nerve motor fibers at the elbow can lead to a loss of dexterity ${ }^{3}$ and a decrease in grip and pinch strength, ${ }^{3}$ both of which can impact performance of functional activities. ${ }^{4}$

As part of a comprehensive assessment for suspected ulnar neuropathy, clinical testing plays an important role in the initial identification of a lesion and determining subsequent changes from the patient's baseline status. With respect to the initial identification of ulnar neuropathy, many tests have demonstrated high degrees of diagnostic value which can be estimated by comparing dichotomous clinical test outcomes to gold standard outcomes. These statistical estimations, delineated in Figure 1, are termed sensitivity, specificity, and positive and negative predictive values. ${ }^{5}$ Sensitivity represents the probability that a positive clinical test outcome correctly identifies the presence of ulnar neuropathy (according to an electrodiagnostic gold standard), whereas specificity represents the probability that a negative clinical test outcome correctly identifies the absence of ulnar neuropathy according to a gold standard. ${ }^{5}$ The positive predictive value represents the probability of disease among patients with a positive clinical test outcome. ${ }^{5}$ The negative predictive 
Calculating Statistics of Measurement Validity

\begin{tabular}{|c|c|c|c|}
\hline \multirow{4}{*}{ 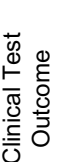 } & & \multicolumn{2}{|c|}{ Gold Standard Results } \\
\hline & & Disease Present & Disease Absent \\
\hline & Positive & A & B \\
\hline & Negative & $\mathrm{C}$ & $\mathrm{D}$ \\
\hline
\end{tabular}

Statistics

-Sensitivity $=A /(A+C)$

-Specificity $=D /(B+D)$

-Positive Predictive Value= $A /(A+B)$

$\begin{array}{ll}- \text { Negative Predictive Value }=D /(C+D) & D=\text { True negative test outcome }\end{array}$

FIGURE 1. Calculating Statistics of Measurement Validity.

value represents the probability of no disease among patients of patents with a negative clinical test outcome. ${ }^{5}$ Sensitivity, specificity, positive predictive value, and negative predictive value can be derived from a single research study and are delineated in Figure 2. Regardless of the test outcome, the examiner must always remain vigilant for other factors which may contribute to the patient's symptoms or alter the expected clinical presentation. Some of these circumstances described in the literature include a double or multiple crush syndrome, ${ }^{6}$ forearm anastamosis, metabolic disorders, cervical spine disorders affecting the C8-T1 nerve roots, brachial plexopathies, central neurological disorders, extraneural soft tissue masses, history of trauma, and congenital anomalies. ${ }^{7,8}$

Two essential aspects of ulnar neuropathy assessment relate to the consistency of clinical test administration and the accurate identification of signs or compensatory movements. If clinical tests are consistently administered properly, the hand therapist can have more confidence in identifying the presence of a lesion, locating the entrapment site (typically occurring either at the elbow or the wrist), ${ }^{8}$ and estimating the extent of motor involvement, especially in the absence of clinically visible muscle atrophy. The accurate identification of test outcomes also guides the hand therapist's selection of therapeutic interventions and provides important information to relay to the physician in the case of a questionable diagnosis. Thus, the purpose of this article is to review ulnar nerve provocative testing and the substantial collection of diagnostic signs and tests found in the literature. It is noteworthy that a comprehensive evaluation of the ulnar nerve should include sensibility testing, which has been thoroughly discussed by Novak and Mackinnon in 2005 and will not be included in this review. ${ }^{9}$

\section{OVERVIEW OF ULNAR NERVE PROVOCATIVE TESTING}

The premise underlying provocative testing for upper extremity compression neuropathies is that local ischemia results in axonal hyperexcitability and a lowered threshold to generate an action potential at the entrapment site. ${ }^{10}$ Therefore, even if a patient is not symptomatic, mechanical stimuli to the location of compressed axons can elicit predictable symptoms if sufficient enough to exceed the threshold potential. ${ }^{7}$ Thus, the essence of provocative testing is literally to "provoke" either a sensory or motor response by initiating an ectopic action potential at the site of a compressed nerve. ${ }^{11}$

Most of the literature related to provocative testing of the ulnar nerve involves sensory axons, which are especially useful in identifying the presence of a compression neuropathy without abnormal electrodiagnostic findings or clinically detectable motor unit involvement. ${ }^{12-14}$ Sunderland noted that the sensory fibers supplying the hand and motor fibers innervating the intrinsic muscles are all superficial at the level of the medial epicondyle, while motor fibers innervating the extrinsic flexor carpi ulnaris and FDP (ring and small fingers) are deeper. ${ }^{15}$ Many authors agree that this anatomical arrangement may explain the common clinical finding of hand weakness but infrequent flexor carpi ulnaris and FDP (to the ring and small fingers) weakness in cubital tunnel syndrome. ${ }^{4,} 16$

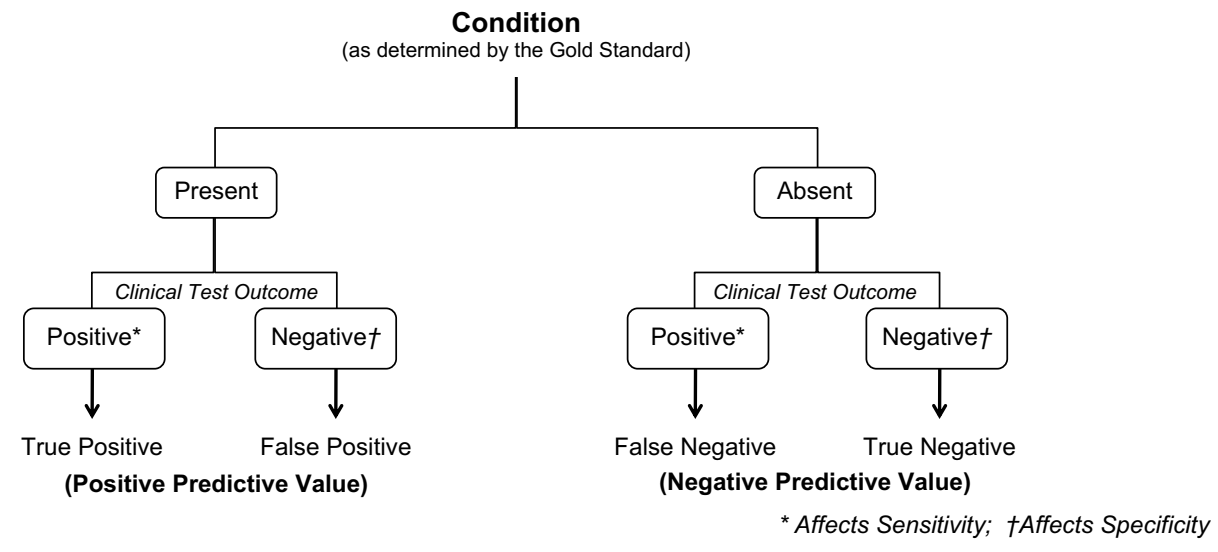

FIGURE 2. Deriving Sensitivity, Specificity, Positive Predictive Value, and Negative Predictive Value from a Research Study. 
This anatomical arrangement may also explain why sensory complaints such as paresthesias are an early finding in ulnar neuropathy at the elbow ${ }^{15}, 17$ as the superficial sensory fibers are often affected before motor fibers. ${ }^{18}$

In some cases, positive provocative testing may be the only evidence of a mild compression neuropathy. ${ }^{9}$ It has also been reported that patients with ulnar nerve dysfunction can present with positive test outcomes but have normal electrodiagnostic findings. ${ }^{1}$ One reason for this discrepancy is that nerve conduction studies selectively stimulate large-diameter myelinated axons, but the ulnar nerve pathology may only reside in the small- and medium-diameter axons, resulting in a normal electrodiagnostic examination even with significant symptomology. ${ }^{19}$ Regardless of the electrodiagnostic findings, results of provocative testing are used for diagnostic purposes, to help assess the severity of ulnar nerve compression and in documenting progression or recovery over a period of time. ${ }^{1,20}$ The following ulnar nerve provocative tests are part of a comprehensive upper extremity evaluation for patients with suspected ulnar nerve compression neuropathy and have been summarized in Table 1.

\section{Spurling's Test}

Plexopathies of the inferior portion of the brachial plexus can result in distal paresthesias similar to an ulnar nerve entrapment at the elbow. They can be caused by a variety of conditions such as cervical disc disease, arthritis, soft tissue tumors (such as a Pancost tumor of the lung), or thoracic outlet syndrome. ${ }^{7}$ Originally termed the "neck compression test,"
Spurling's test is performed by first asking the patient to actively extend and laterally flex the neck to the involved side. ${ }^{21}$ Once the patient has maintained this neck position, the examiner then applies axial compression to the cervical spine as shown in Figure 1. This test is designed to provoke or worsen radicular sensory symptoms at the nerve root level as it exits the foramen. Studies by Tong and colleagues ${ }^{22}$ and Viikari-Juntura and colleagues ${ }^{23}$ agree that the Spurling's test has generally high specificity (93\% and as low as $92 \%$, respectively) but much lower sensitivity ( $30 \%$ and up to $50 \%$, respectively), thus a positive Spurling's test should be interpreted with caution.

\section{Combined Pressure and Flexion Test}

The combined pressure and flexion test, as shown in Figure 2, combines elbow flexion and external pressure to the ulnar nerve. The test is performed with the elbow in maximum flexion with simultaneous examiner-applied external pressure to the ulnar nerve just proximal to the cubital tunnel. A positive test is recorded when the patient reports the presence or worsening of paresthesias in the ulnar nerve distribution. Novak and colleagues investigated the sensitivity and specificity of performing this test after 30 and $60 \mathrm{sec}^{24}$ Sensitivity results were higher $(98 \%)$ when pressure was sustained for $60 \mathrm{sec}$, as compared with $91 \%$ when pressure was sustained for $30 \mathrm{sec}$. Conversely, specificity results were slightly higher when pressure was sustained for $30 \mathrm{sec}(97 \%)$ as compared with $60 \mathrm{sec}(95 \%) .{ }^{24}$ This indicates that although sustaining external pressure for $60 \mathrm{sec}$ can better detect the presence of pathology, there may

TABLE 1. Summary of Provocative Tests for Ulnar Neuropathy

\begin{tabular}{|c|c|c|}
\hline Test Name & Description & Positive Result \\
\hline \multicolumn{3}{|l|}{ Provocative tests for sensory axons } \\
\hline Spurling's test & $\begin{array}{l}\text { Patient actively extends and laterally } \\
\text { flexes neck, then examiner applies } \\
\text { axial compression to cervical spine }\end{array}$ & $\begin{array}{l}\text { Presence/worsening of numbness/ } \\
\text { paresthesias in nerve root distribution }\end{array}$ \\
\hline Combined pressure and flexion test & $\begin{array}{l}\text { Elbow in maximum flexion; the } \\
\text { examiner applies external pressure } \\
\text { just proximal to the cubital tunnel } \\
\text { (typically for } 60 \mathrm{sec} \text { ) }\end{array}$ & $\begin{array}{l}\text { Presence/worsening of numbness/ } \\
\text { paresthesias in the ulnar nerve } \\
\text { distribution }\end{array}$ \\
\hline Elbow flexion test & $\begin{array}{l}\text { Many positions described. Recommend } \\
\text { full active elbow flexion, with forearm } \\
\text { supinated, wrist in neutral. Sustain for } \\
60 \mathrm{sec}\end{array}$ & $\begin{array}{l}\text { Presence/worsening of numbness/ } \\
\text { paresthesias in the ulnar nerve } \\
\text { distribution }\end{array}$ \\
\hline Pressure test & $\begin{array}{l}\text { Elbow in } 20^{\circ} \text { of flexion, forearm in } \\
\text { supination. Examiner applies external } \\
\text { pressure just proximal to the cubital } \\
\text { tunnel for } 60 \mathrm{sec}\end{array}$ & $\begin{array}{l}\text { Presence/worsening of numbness/ } \\
\text { paresthesias in the ulnar nerve } \\
\text { distribution }\end{array}$ \\
\hline Tinel's sign & $\begin{array}{l}\text { Mechanical percussion along the course } \\
\text { of the ulnar nerve }\end{array}$ & $\begin{array}{l}\text { Patient reports tingling, paresthesias, or } \\
\text { a "shocking" sensation in the nerve's } \\
\text { sensory distribution }\end{array}$ \\
\hline \multicolumn{3}{|l|}{ Provocative tests for motor axons } \\
\hline Motor Tinel's sign & $\begin{array}{l}\text { Mechanical percussion along the course } \\
\text { of the ulnar nerve }\end{array}$ & $\begin{array}{l}\text { Visible "motor jerk" in addition to } \\
\text { paresthesias of ulnar nerve- } \\
\text { innervated muscles }\end{array}$ \\
\hline
\end{tabular}


be slightly more false-positive responses at the 60 -sec interval as compared with the 30 -sec interval.

\section{Elbow Flexion Test}

The elbow flexion test also assesses the ectopic activity of sensory axons and the specific test position has been described in a variety of ways. Buehler and Thayer evaluated subjects with the elbows actively "fully but not forcefully flexed" and the wrist in full extension for a period of up to $180 \mathrm{sec}$, documenting pain, numbness, tingling, location, and symptom severity. ${ }^{25}$ Wadsworth tested patients in full active elbow flexion for $5 \mathrm{~min}$ with no mention of the forearm, wrist, or shoulder positions. ${ }^{26}$ Rayan and colleagues examined four different versions of the elbow flexion test on 204 elbows in 102 normal subjects, all of which positioned the elbow in 90 degrees of flexion with different wrist (wrist flexion and extension) and shoulder positions (either neutral or 90 degrees of abduction). ${ }^{27}$ Regardless of testing position or length of time the test is sustained, a positive elbow flexion test is considered one which increases the patient's numbness or paresthesias in the ulnar nerve distribution. ${ }^{28}$

Reliability and validity studies for the elbow flexion test have been performed using different amounts of time and different upper extremity positions. A clinical validity study by Rosati and colleagues ${ }^{29}$ evaluated normal subjects performing all four elbow flexion tests described in a study by Rayan and colleagues. ${ }^{27}$ In Rayan's study, $3.6 \%$ of subjects had a positive test at $1 \mathrm{~min}$ and $16.2 \%$ were positive at 3 minutes. The authors concluded that sustaining the elbow flexion test for $1 \mathrm{~min}$ would reduce the number of false positives in normal individuals. Rosati's study reported that more positive results were associated with the passing of time, but the effect of specific test position was not investigated. ${ }^{29}$ Novak and colleagues reported a sensitivity of $32 \%$ for the elbow flexion test after $30 \mathrm{sec}$ and $75 \%$ after $60 \mathrm{sec}$, while specificity was $99 \%$ for both 30 and $60 \mathrm{sec}^{24}$ For this study, the subject's elbow was placed in maximum flexion with full supination and the wrist in neutral. These studies indicate that the most appropriate duration of the elbow flexion test is $60 \mathrm{sec}$ which will minimize the number of false positive results yet still be sensitive enough to detect ulnar nerve pathology. The aforementioned studies used a minimum of 90 degrees of elbow flexion when performing the test; however, the effect the wrist and forearm position was not specifically investigated.

\section{Pressure Test}

The "pressure test" or "pressure-provocative test" originally described by Paley and McMurtry ${ }^{30}$ as part of the carpal tunnel syndrome literature has been applied to assess sensory axons of the ulnar nerve. ${ }^{24}$ Consistent with the underlying principle of provocation, external pressure at the compression site can generate ectopic action potentials in sensory axons with subsequent paresthesias. The pressure test (shown in Figure 3) as described by Novak and colleagues for the ulnar nerve is performed by applying direct pressure immediately proximal to the cubital tunnel with the elbow in 20 degrees of flexion and the forearm in supination for $60 \mathrm{sec} .{ }^{24}$ A positive test is annotated with the reproduction of the patient's paresthesias in the ulnar nerve distribution. These researchers report a sensitivity of $55 \%$ after 30 of pressure, $89 \%$ after $60 \mathrm{sec}$ of pressure, and a specificity of $98 \%$ for both 30 and 60 sec of pressure. These sensitivity findings are higher than those for the elbow flexion test and the specificity findings are slightly lower.

\section{Tinel's Sign}

The presence of a Tinel's sign ${ }^{31}$ can help assess the status of sensory axons in many upper extremity compression neuropathies, including those involving the ulnar nerve. In 1915, Jules Tinel was the first to describe the "signe de fourmillent" as a tingling sensation in the distribution of sensory or mixed peripheral nerves after percussion over the site of newly formed axons. ${ }^{31}$ A positive Tinel's sign has been reported in patients with compression neuropathies, with partial or complete nerve lacerations, as well as in the presence of a neuroma. ${ }^{15}$ Novak and Mackinnon recommend assessing for a Tinel's sign along the course of the ulnar nerve beginning proximal to the cubital tunnel and progressing distally through the cubital tunnel to ensure that all possible entrapment sites at the elbow are considered. ${ }^{9}$ Assessing for the presence of a Tinel's sign should

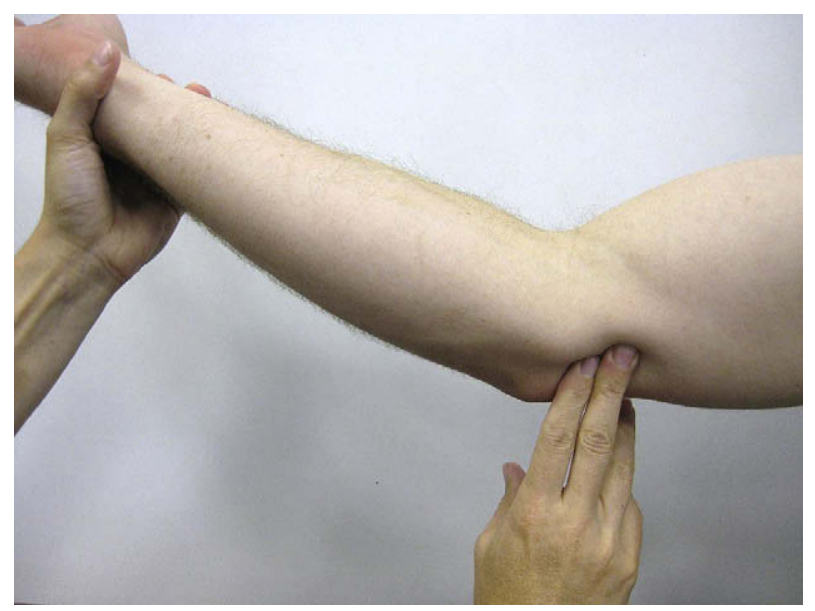

FIGURE 3. Pressure test. The forearm is supinated and the elbow is flexed to $20^{\circ}$. The examiner applies pressure just proximal to the cubital tunnel for $60 \mathrm{sec}$. 
also be performed along other potential sources of ulnar nerve compression to include distal sites around Guyon's canal, as well as proximal sites to include the supra and infraclavicular areas. ${ }^{7}$

One of the most important clinical aspects of the Tinel's sign relates to nerve regeneration as evidenced by distal progression of a Tinel's sign over time. In 1946, Nathan and Rennie were the first to document this essential feature of the Tinel's sign: whether or not it is progressive. ${ }^{32}$ Napier suggested that a Tinel's sign that progresses distally is strong evidence of continuity of the nerve and that the rate of descent can be useful in predicting the degree of recovery. ${ }^{33}$ Napier's conclusions have been challenged by other authors and the usefulness of the Tinel's sign has been scrutinized in the literature. As early as 1919, Stookey concluded that a positive Tinel's sign is not necessarily indicative of an incomplete nerve lesion. ${ }^{34}$ Seddon and colleagues reported it to be irregular and unreliable as a guide to both the completeness of a lesion and the rate of regeneration..$^{35}$ Nonetheless, documentation of a Tinel's sign is commonly performed in many surgical and rehabilitation practices for both diagnostic and recovery purposes. More empirical studies are needed to quantify the relationship between a progressing Tinel's sign and the degree of recovery.

Research studies have evaluated the usefulness of the Tinel's sign in cubital tunnel syndrome. A recent reliability and validity study by Novak and colleagues reported a $70 \%$ sensitivity and $98 \%$ specificity for the Tinel's sign. ${ }^{24}$ Compounding this question of diagnostic usefulness, a study by Rayan and colleagues revealed more substantial false positives in studies using normal subjects. These researchers found a positive Tinel's sign at the cubital tunnel in $23.5 \%$ of 200 elbows of normal subjects, $50 \%$ of which were bilateral. ${ }^{27}$ Examiner technique may help minimize a false-positive result; for instance, McPherson and Meals advise against vigorous percussion and recommend gentle tapping with the index finger to elicit a Tinel's sign. ${ }^{17}$ The aforementioned controversy suggests that the presence of an appropriately performed Tinel's sign may be most useful clinically in combination with other identified findings of nerve compression. ${ }^{8}$

\section{Motor Tinel's Sign}

The only provocative test specifically addressing motor axons is the motor Tinel's sign, which was first described by Montagna and Liguroi. ${ }^{36}$ In a motor Tinel's sign, percussion of the ulnar nerve results in both the sensory response of paresthesias and a visible "motor jerk" of ulnar nerve-innervated muscles. The hallmark of a positive motor Tinel's sign is the presence of the involuntary motor response, which is not seen in the original Tinel's sign which assesses the presence of a sensory response. Kingery and colleagues investigated 50 cases of ulnar neuropathy and reported a sensitivity of $78 \%$ and a specificity of $79 \%$ for the motor Tinel's sign. ${ }^{37}$

\section{OVERVIEW OF ULNAR NERVE MOTOR SIGNS AND TESTS}

There are a greater number of signs and tests that have been described to identify the presence of ulnar nerve motor dysfunction than any other upper extremity compression neuropathy, the first being described by Duchenne in $1867 .{ }^{38}$ Although these signs and tests identify the presence of ulnar nerve motor dysfunction, there have been few empirical studies quantifying the relationship between the dichotomous outcomes and the extent of pathology. The following ulnar nerve motor signs and tests have been grouped according to the affected musculature and have been summarized in Table 2.

\section{Motor Signs Involving the Adductor Pollicis}

\section{Froment's Sign}

In 1915, Froment reported a motor sign of ulnar neuropathy that assesses the method of lateral pinch between the thumb and radial side of the index finger. ${ }^{39}$ A positive sign (as shown in Figure 4) is characterized by flexion of the interphalangeal (IP) joint of the thumb by the anterior interosseous nerve-innervated flexor pollicis longus to substitute for a weak ulnar nerve-innervated adductor pollicis during a lateral pinching task using a piece of paper. The presence of Froment's sign is considered to be an indicator of ulnar nerve motor dysfunction; however, Sunderland cautions that this sign is unreliable unless the examiner monitors the "trick action" of the extensor pollicis longus that allows the patient to stabilize the paper without IP joint flexion. ${ }^{15} \mathrm{~A}$ few authors have emphasized the action of the extensor pollicis longus as a supplementary thumb adductor when assessing for a Froment's sign. ${ }^{40-42}$ Mannerfelt noted that the ability of the extensor pollicis longus to act as a thumb adductor decreases with wrist flexion. ${ }^{43}$ Thus when assessing for a Froment's sign, it may be beneficial to have the wrist in slight flexion.

\section{Jeanne's Sign}

A few months later in 1915, Jeanne published a different substitution pattern for a weak adductor pollicis muscle using Froment's lateral pinch task. ${ }^{44}$ Eventually termed "Jeanne's sign," the patient hyperextends the metacarpophalangeal joint of the thumb to stabilize a piece of paper or thin object during lateral pinch. A positive Jeanne's sign is 


\begin{tabular}{ll} 
Test Name & \multicolumn{1}{c}{ Description } \\
$\begin{array}{l}\text { Motor signs involving the adductor pollicis } \\
\text { Froment's sign }\end{array}$ & $\begin{array}{l}\text { The patient holds a piece of paper using } \\
\text { a lateral pinch. The examiner then pulls } \\
\text { the paper distally along the thumb's } \\
\text { longitudinal axis and assesses the } \\
\text { patient's method of stabilization } \\
\text { The patient holds a piece of paper using } \\
\text { a lateral pinch. The examiner then pulls } \\
\text { the paper distally along the thumb's } \\
\text { longitudinal axis and assesses the } \\
\text { patient's method of stabilization }\end{array}$
\end{tabular}

Motor signs and tests involving the interossei

First DI screening test

The patient places the radial aspects of the index fingers together in abduction and then pushes them together using only the index fingers.

Finger flexion sign Performed bilaterally at the same time. Both forearms and wrists are in neutral. Examiner first places a piece of paper between the middle and ring fingers in both hands and then pulls the paper distally.

Crossed finger test

Egawa's sign Examiner asks the patient to cross the middle finger over the index finger Examiner then asks the patient to flex the middle finger MP joint and then to abduct it to both sides. This can be difficult to perform; therefore, bilateral assessment is recommended.

Motor signs involving the ulnar nerve-innervated lumbricals

Duchenne's sign

Sign is identified by observing the posture of the small and ring fingers on the involved side.

André-Thomas sign Sign identified by observing the compensatory pattern used in the ring and small fingers during actions involving EDC use.

Motor signs involving the hypothenar musculature

Wartenberg's sign

Patient actively abducts the fingers with the forearm in pronation and the wrist in neutral. Observe the small finger's ability to fully adduct.

Masse's sign Observe the metacarpal arch as compared with the uninvolved side. The convex nature of the ulnar aspect of the hand is altered due to hypothenar atrophy.

Pitres-Testut sign

Noted after the examiner asks the patient to shape the hand in the form of a cone. Although present in the literature, this sign is not commonly used in clinical practice settings.

Palmaris Brevis sign A rarely observed sign in lower ulnar nerve palsy where the lesion selectively affects the deep branch. Determine the presence of this sign by observing and evaluating the palmaris brevis muscle as compared with the uninvolved side.

Motor signs involving the extrinsic ulnar nerve-innervated muscles Nail file sign

Patient attempts to make a hook fist. Examiner places an index finger along the volar surface of the patient's small and ring fingers, leaving the DIPs free to contract.
Thumb IP flexion compensates for a weak adductor pollicis muscle

Thumb MP hyperextension compensates for a weak adductor pollicis muscle

The involved side will be overpowered by the uninvolved side and pushed into adduction

The involved side will use MP flexion to compensate for interossei weakness

Inability to cross the fingers. Compare with uninvolved side

Inability to perform this action as compared with uninvolved side

Clawing posture (MP hyperextension and IP flexion) present in the ring and small fingers

Wrist tends to flex with ring and small finger EDC activation

Inability of the small finger to fully adduct and touch the ring finger. Compare with the uninvolved side

Flattened metacarpal arch

Inability to shape the hand in the form of a cone

The sparing of the palmaris brevis muscle as compared with the uninvolved side

Decreased small and ring finger FDP strength as compared with the uninvolved side

$\mathrm{MP}=$ metacarpophalangeal; $\mathrm{IP}=$ interphalangeal; $\mathrm{EDC}=$ extensor digitorum communis; $\mathrm{DIP}=$ distal interphalangeal; FDP = flexor digitorum profundus. 

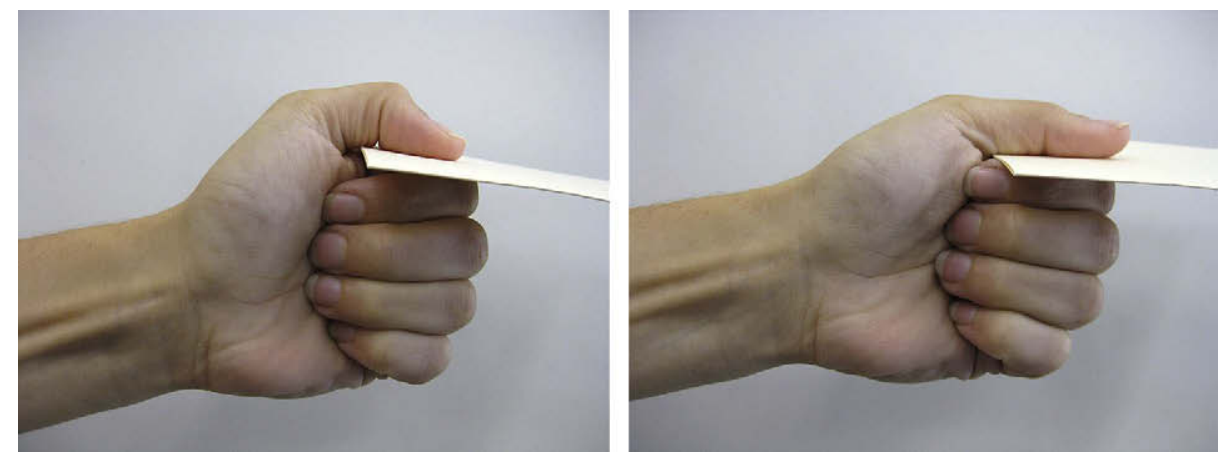

FIGURE 4. Positive Froment's sign on the left, negative Froment's sign on the right. It is recommended that the wrist be positioned in slight flexion when performing this test.

not commonly seen in patients with ulnar neuropathy as compared with a positive Froment's sign; however, patients with ulnar neuropathy can gradually develop thumb metacarpophalangeal laxity through substitution patterns for a weak adductor pollicis. The presence of a Jeanne's sign and a Froment's sign are mutually exclusive during a single test.

\section{Motor Signs and Tests Involving the Interossei}

Tests evaluating interossei motor function, especially the first dorsal interosseous muscle, are another important part of the motor assessment. Because the first dorsal interosseous muscle can be the last muscle innervated by the ulnar nerve, its function is important in detecting initial motor axon involvement. McPherson and Meals recommend a screening test for unilateral ulnar nerve lesions by comparing first dorsal interosseous muscle strength with the uninvolved side. ${ }^{17}$ This screening test, designed to mitigate the examiner's subjective assessment of muscle strength, involves placing the radial aspects of the index fingers together in abduction and asking the patient to push them together. If the involved index finger can be overpowered by the uninvolved side and pushed into an adducted position, then first dorsal interosseous weakness is suspected. A unilateral test of first dorsal interosseous strength involves resisting index finger radial abduction. ${ }^{17}$

\section{Finger Flexion Sign}

Tsujino and Macnicol described a test to identify the the "finger flexion sign" for ulnar neuropathy that is part of an assessment of interossei function. ${ }^{45}$ This test, which is performed on both hands at the same time, assesses interosseous muscle function by asking the patient to hold a sheet of paper between the middle and ring fingers while the examiner pulls it distally along the longitudinal axis of the radius. A positive outcome (shown in Figure 5) is noted with metacarpal-phalangeal flexion in the affected side as the extrinsic finger flexors try to compensate for interossei weakness. The authors contend that this test can detect muscle weakness in the early stage of ulnar neuropathy. This test is based on Sunderland's finding that the palmar interossei are functioning if a thin object can be held about the ring finger without any digital flexion. ${ }^{15}$

\section{Crossed Finger Test}

The crossed finger test (shown in Figure 6) has been described by Earle and Vlastou as another test of interossei function. ${ }^{46} \mathrm{~A}$ positive crossed finger test is noted with the inability to completely cross the middle finger over the extended index finger, representing weakness in the first volar interosseous and second dorsal interosseous muscles. Earle indicated that a negative crossed finger test is recorded if the patient can cross the fingers normally. Consistent with Sunderland's findings, Earle also identified that patients may use the extensor indicis proprius to initiate the crossing finger movement by placing the nail of the index finger under the pad of the middle finger. ${ }^{46}$ The crossed finger test can be used as a screening test for patients with acute ulnar nerve damage, but more empirical studies are needed to assess the value of this test.

$$
\text { Egawa's Sign }
$$

Assessment of the long finger's ability to abduct has also received considerable attention in the literature. The inability of the third digit to abduct in either direction was one of the tests described in 1925 by Pitres and Testut. ${ }^{43}$ Many authors have expressed concern that some abduction can still be initiated by the digital extensors even with full dorsal interossei paralysis. ${ }^{47-49}$ This substitution can be prevented by first stabilizing the wrist and ensuring metacarpophalangeal joint flexion before assessing long finger abduction. ${ }^{15}$ This is part of the biomechanical basis for 

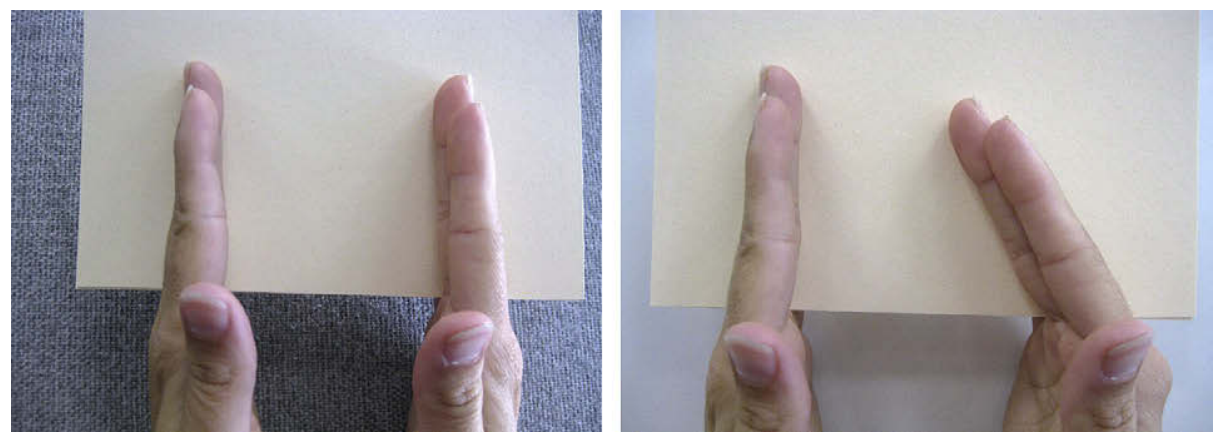

FIGURE 5. A positive finger flexion sign is present in the right hand on the right photo. The left photo shows a negative finger flexion sign as the paper is pulled distally.

Egawa's sign. ${ }^{50}$ To perform this test, the patient flexes the metacarpophalangeal joint and subsequently attempts radial and ulnar abduction of the long finger (as shown in Figure 7); failure to do so is considered a positive Egawa's sign.

\section{Motor Signs Involving the Ulnar Nerve-Innervated Lumbricals}

\section{Duchenne's Sign}

There are few signs in the literature addressing the function of the ulnar nerve-innervated lumbrical muscles. Duchenne was the first to describe changes of the small and ring fingers with the metacarpophalangeal joints positioned in hyperextension and IP joints in flexion in patients with ulnar neuropathy. ${ }^{38}$ Known as "Duchenne's Sign," clawing of the ring and small fingers is a result of lumbrical weakness and unopposed action of the extrinsic finger extensors. This sign is more pronounced in a low ulnar nerve injury as the FDP is intact and contributes to increased IP flexion.

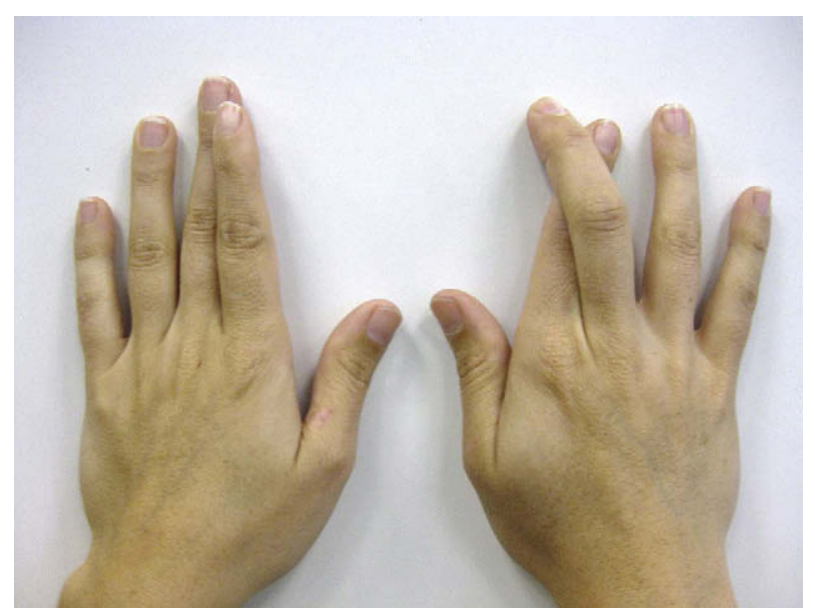

FIGURE 6. Crossed finger test. The left hand shows a positive crossed finger test as the index finger is unable to completely cross over the middle finger. The right hand shows a negative crossed finger test.

\section{André-Thomas Sign}

With a lack of phalangeal extension from the ulnar nerve-innervated lumbricals, substitution patterns will emerge as signs of ulnar nerve dysfunction. In 1974, Aiche and Delahi described a "pure sign of lumbrical function" through active metacarpophalangeal flexion when the IP joints are held in full extension. ${ }^{51}$ The researchers noted the importance of distinguishing between the action of the lumbricals from motion resulting from the extrinsic digital extensors or interossei. Sunderland ${ }^{52}$ explained that the extensor digitorum communis (EDC) can assist with phalangeal extension; however, in the case of advanced ulnar neuropathy with the presence of metacarpalphalangeal hyperextension, the mechanical disadvantage of the EDC results in extrinsic digital flexor compensation. ${ }^{15}$ This is the biomechanical basis for the André-Thomas sign, described as the tendency of the wrist to flex during action of extrinsic digital extensors in patients with ulnar neuropathy. ${ }^{43}$

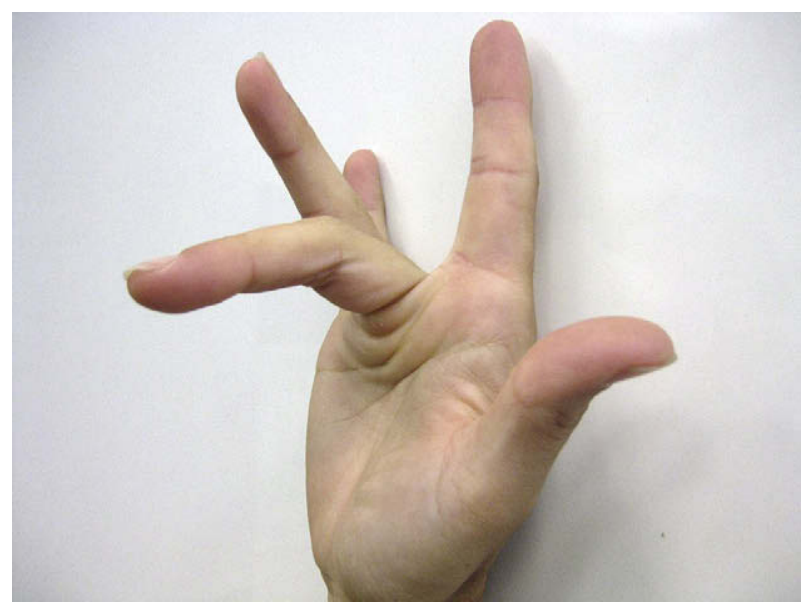

FIGURE 7. Testing for Egawa's sign. Patient first flexes the metacarpophalangeal joint and then abducts the finger in both directions. Because this maneuver can be difficult to perform, comparison with the uninvolved side is strongly recommended. 


\section{Motor Signs and Tests Involving the Hypothenar Musculature}

$$
\text { Wartenberg's Sign }
$$

Motor function of the hypothenar eminence and its contribution to thumb opposition has received considerable attention in identifying ulnar nerve motor dysfunction. In particular, analysis of small finger abduction has resulted in some signs of ulnar neuropathy. Duchenne ${ }^{38}$ highlighted this clinical observation in his book, originally published in 1867, noting that "the impossibility to adduct the extended fingers represents the first step of paralysis of the interossei." In 1939, Wartenberg published a sign of ulnar neuropathy ${ }^{53}$ that has since been coined "Wartenberg's Sign." He described case studies whereby the small finger assumed a "position of abduction," especially when the fingers are held extension. Wartenberg emphasized that this sign represents a late manifestation of ulnar nerve palsy. ${ }^{53}$ Procedures to assess for the presence of a Wartenberg's sign involve asking the patient to first fully pronate and maintain the wrist in a neutral position. Then, the examiner asks the patient to first fully abduct and fully adduct the fingers. A positive Wartenberg's sign is recorded if the small finger cannot fully adduct to touch the ulnar border of the ring finger (as shown in Figure 8). Comparison to the uninvolved side can also be helpful in identifying abductor digiti minimi weakness.

McPherson and Meals describe a screening test for the abductor digiti minimi through comparison to the uninvolved side. ${ }^{17}$ This screening test is performed with the patient's forearms supinated and the tips of both small fingers resisting each other in an effort to identify if one side can overpower the other. McPherson and Meals also point out that this screening test can mitigate the examiner's subjective assessment of strength. ${ }^{17}$

\section{Masse's Sign and Pitres-Testut's Sign}

Chronic ulnar nerve injury involving the motor component of the hypothenar muscles are the inspiration for the following signs that reflect changes in appearance and the shape of the hand. In 1916, Masse reported a flattened metacarpal arch as a sign of ulnar neuropathy, later termed Masse's sign. ${ }^{43}$ Sunderland suggested that atrophy of the hypothenar muscles contribute to the loss of convex nature of the ulnar aspect of the hand, ${ }^{15}$ although interossei atrophy clearly contributes to this sign as well. Pitres-Testut described another ulnar nerve sign as the inability to shape the hand in the form of a cone, ${ }^{43}$ which can be partially attributed to hypothenar atrophy. Both of these signs are present with significant atrophy, are more difficult to detect, and are not as commonly administered in clinical practice. There has not been an empirical

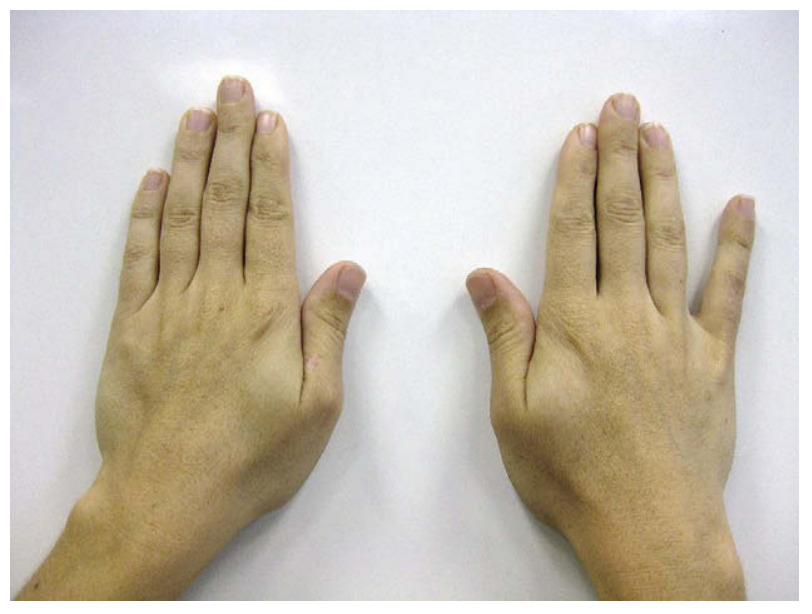

FIGURE 8. Wartenberg's sign. The right hand indicates a positive Wartenberg's sign because the small finger is unable to fully adduct and touch the ring finger. The left hand indicates a negative Wartenberg's sign.

study to determine the relative frequency of these two signs in patients with ulnar neuropathy.

\section{Other Noteworthy Hypothenar Observations}

Other motor considerations surrounding hypothenar function and its contribution to opposition have been documented in conjunction with ulnar neuropathy. Sunderland showed that decreased motor function of the small finger intrinsic muscles results in incomplete thumb opposition. ${ }^{54}$ This incomplete opposition is due to small finger metacarpophalangeal hyperextension and abduction and IP flexion. Paralysis of these muscles also leads to a lack of hypothenar elevation which has also been cited by Sunderland as a contributing factor to incomplete thumb opposition. In 1961, Mumenthaler reported a lack of a typical "dimple" in the hypothenar eminence due to atrophy of the palmaris brevis muscle. ${ }^{55}$ In lower ulnar nerve lesions, the palmaris brevis can be spared when the pathology selectively affects the deep branch of the ulnar nerve, ${ }^{7}$ referred to as the palmaris brevis sign. ${ }^{56}$ All of these observations are more subtle, and formal testing procedures have not been identified or empirically evaluated.

\section{Motor Signs Involving Extrinsic Ulnar Nerve-Innervated Muscles}

Involvement of extrinsic ulnar nerve-innervated muscles, namely the flexor carpi ulnaris and FDP to the small and ring fingers, can occur with longstanding ulnar nerve compression occurring at the elbow. It is unusual to identify pronounced wrist flexion weakness in patients with advanced ulnar neuropathy because of the strong influence of the 
flexor carpi radialis and the palmaris longus, if present. These muscles can more than adequately compensate for extensor carpi ulnaris weakness. Conversely, weakness of the ulnar nerve-innervated portion of the FDP can result in functional deficits, most notable of which is decreased grip strength. ${ }^{57}$

\section{Nail File Sign}

With regard to specific tests in the literature involving the FDP, in 1919 Pollock described the inability to flex the distal phalanx on the small finger in patients with ulnar neuropathy. ${ }^{48}$ More recently, in 1999, Kapandji identified and coined the term "nail file sign" as another sign of unilateral ulnar neuropathy. ${ }^{58}$ The inspiration for the name of this sign came from a patient who complained of the inability to file the nail of her small finger. To assess for the presence of this sign, the patient attempts to make a hook fist while the examiner's index finger is placed along the volar surface of the patient's ring and small fingers, leaving the distal interphalangeal (DIP) joints free to contract (see Figure 9). The examiner assesses the strength of the FDP by trying to extend the DIP joints of the ring and small fingers while the patient activates the profundus. This maneuver compares the strength of the FDP on the involved side to that of the uninvolved side. A positive nail file sign represents identified weakness in the ulnar nerve-innervated portion of the FDP and an indicator of a possible higher level ulnar nerve lesion. ${ }^{58}$

\section{CONCLUSION}

Provocative testing and assessment of motor function are two important aspects of a comprehensive

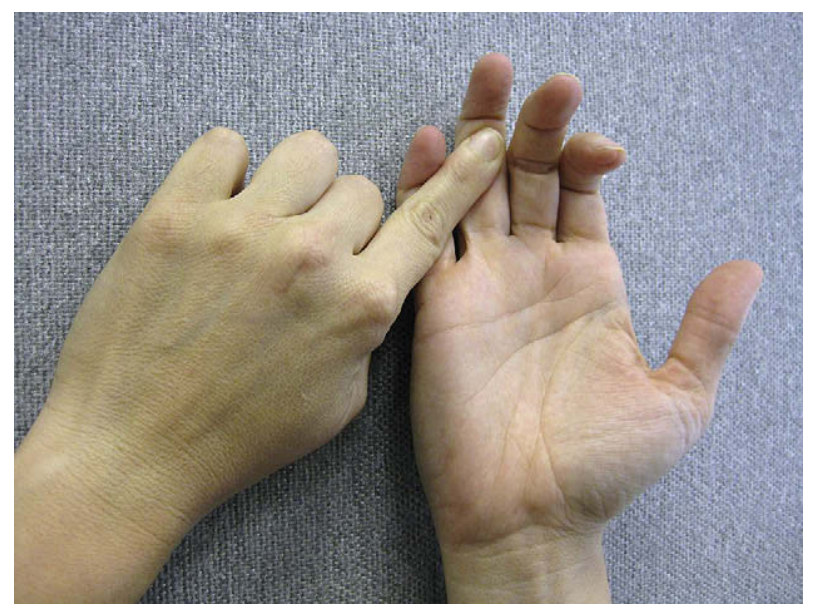

FIGURE 9. Testing for the nail file sign. The distal interphalangeal joints of the ring and small fingers are allowed to flex, blocking the proximal interphalangeal joints in extension. The general strength is assessed and compared with the uninvolved side. This sign may be present in severe compression of ulnar nerve motor axons at the elbow. upper extremity assessment for patients suspected with ulnar neuropathy. Results of this testing can help confirm the diagnosis, rule out other potential sources of pathology, or provide evidence of recovery progress after an initial baseline has been established. It is important that the hand therapist administer the testing procedures in a consistent manner and understand what constitutes the presence of a positive or negative sign. Evaluating the uninvolved side can be helpful, especially for some of the more subtle motor signs and tests. As with other aspects of patient assessment, the clinical tests discussed in this narrative review article constitute only one aspect of the examination and should not substitute for other key components, such as taking a thorough medical and occupational history. Empirical research studies are indicated to further quantify the relationship between the outcomes of ulnar nerve motor tests and the severity of the lesion. Additionally, studies are needed to determine the most robust motor signs and quantify the likelihood of those that are more likely to be seen in the early stages of the disease.

\section{Acknowledgments}

The authors would like to thank Amanda Antczak for her assistance in preparing the article and Dr. Richard Curtis in Orange Park, FL, for his mentorship.

\section{REFERENCES}

1. Bradshaw D, Shefner JM. Ulnar neuropathy at the elbow. Neurol Clin. 1999;17:447-61.

2. Layzer RB. The origin of muscle fasciculations and cramps. Muscle Nerve. 1994;17(11):1243-9.

3. Folberg C, Weiss PC, Akelman E. Cubital tunnel syndrome: part I. presentation and diagnosis. Orthop Rev. 1994;23:136-44.

4. Bozentka D. Cubital tunnel syndrome pathophysiology. Clin Orthop Rel Res. 1998;351:90-4.

5. Grimm L, Arnold P (eds). Reading and Understanding Multivariate Statistics. Washington, DC: American Psychological Association, 1995.

6. Mackinnon S. Double and multiple "crush" syndromes. Hand Clin. 1992;8(2):369-90.

7. Idler R. General principles of patient evaluation and nonoperative management of cubital tunnel syndrome. Hand Clin. 1996;12(2):397-403.

8. Rayan G. Proximal ulnar nerve compression: cubital tunnel syndrome. Hand Clin. 1992;8(2):325-36.

9. Novak C, Mackinnon SE. Evaluation of nerve injury and nerve compression in the upper quadrant. J Hand Ther. 2005;18: 230-40.

10. Mackinnon S. Pathophysiology of nerve compression. Hand Clin. 2002;18:231-41.

11. Caress J, Walker FO. The spectrum of ectopic motor nerve behavior: From fasciculations to neuromyotonia. Neurologist. 2002;8:41-6.

12. Cutts S. Cubital tunnel syndrome. Postgrad Med J. 2007;83: 28-31.

13. Greenwald D, Moffitt M, Cooper B. Effective surgical treatment of cubital tunnel syndrome based on provocative clinical testing without electrodiagnostics. Plast Reconstr Surg. 1999; 104:215-8. 
14. Tomaino M, Brach PJ, Vansickle DP. The rationale for and efficacy of surgical intervention for electrodiagnostic-negative cubital tunnel syndrome. J Hand Surg [Am]. 2001;26A:1077-81.

15. Sunderland S. Nerves and Nerve Injuries. New York: Churchill Livingstone, 1978.

16. Apfelberg D, Larson SJ. Dynamic anatomy of the ulnar nerve at the elbow. Plast Reconstr Surg. 1973;51:76-81.

17. McPherson S, Meals RA. Cubital tunnel syndrome. Orthop Clin North Am. 1992;23:111-23.

18. Payan J. Electrophysiological localization of ulnar nerve lesions. J Neurol Neurosurg Psychiatr. 1969;32:208-20.

19. Hilburn J. General principles and use of electrodiagnostic studies in carpal and cubital tunnel syndromes: with special attention to pitfalls and interpretation. Hand Clin. 1996;12:205-21.

20. Adams J, Steinmann SP. Nerve injuries about the elbow. J Hand Surg [Am]. 2006;31A:303-13.

21. Spurling R, Scoville WB. Lateral rupture of the cervical intervertebral discs: a common cause of shoulder and arm pain. Surg Gynecol Obstet. 1944;78:350-8.

22. Tong HC, Haig AJ, Yamakawa K. The Spurling test and cervical radiculopathy. Spine. 2002;27(2):156-9.

23. Viikari-Juntura E, Porras M, Laasonen EM. Validity of clinical tests in the diagnosis of root compression in cervical disc disease. Spine. 1989;14(3):253-7.

24. Novak C, Lee GW, Mackinnon SE, Lay L. Provocative testing for cubital tunnel syndrome. J Hand Surg [Am]. 1994;19A: 817-20.

25. Buehler M, Thayer DT. The elbow flexion test. A clinical test for the cubital tunnel syndrome. Clin Orthop Rel Res. 1988;233: 213-6.

26. Wadsworth T. The external compression syndrome of the ulnar nerve at the cubital tunnel. Clin Orthop. 1977;124:189-204.

27. Rayan G, Jensen CJ, Duke JD. Elbow flexion test in the normal population. J Hand Surg [Am]. 1992;17:86-9.

28. Eversmann W. Compression and entrapment neuropathies of the upper extremity. J Hand Surg [Am]. 1983;8:759-66.

29. Rosati M, Martignoni R, Spagnolli G, Nesti C, Lisanti M. Clinical validity of the elbow flexion test for the diagnosis of ulnar nerve compression at the cubital tunnel. Acta Ortho Belg. 1998; 64(4):366-70.

30. Paley D, McMurtry RY. Median nerve compression test in carpal tunnel syndrome diagnosis reproduces signs and symptoms in affected wrist. Orthop Rev. 1985;14:41-5.

31. Tinel J. Le signed $u$ fourmillement dans les lesions des nerfs peripheriques. Presse Med. 1915;23:388-9.

32. Nathan P, Rennie AM. Lancet. 1946;1:610.

33. Napier J. The significance of Tinel's sign in peripheral nerve injuries. Brain. 1949;72:63-82.

34. Stookey B. Neurol Bull. 1919;2:380.

35. Seddon $\mathrm{H}$, Medawar PB, Smith $\mathrm{H}$. The rate of regeneration of peripheral nerves in man. J Physiol. 1943;102:191-215.
36. Montagna P, Liguori R. The motor Tinel sign: a useful sign in entrapment neuropathy? Muscle Nerve. 2000;23:976-8.

37. Kingery W, Park KS, Wu PB, Date ES. Electromyographic motor Tinel's sign in ulnar mononeuropathies at the elbow. Am J Phys Med Rehabil. 1995;74:419-26.

38. Duchenne G. Physiology of Motion: Demonstrated by Means of Electrical Stimulation and Clinical Observation Applied to the Study of Paralysis and Deformities. Philadelphia: WB Saunders, 1959.

39. Froment J. La prehension dans les paralysies du nerf cubital et Le Signe du Pouce. Presse Med. 1915;23:409.

40. Babinski J, Froment J. Les signes objectifs de la paralysie de l'adducteur du pouce. Revue Neurol [Paris]. 1918;1:484.

41. Kaplan E. Functional and Surgical Anatomy of the Hand. Philadelphia: J.B. Lippincott Company, 1965.

42. Tinel J. Nerve Wounds. London: Baillière, Tindale \& Cox, 1917.

43. Mannerfelt L. Studies on the hand in ulnar nerve paralysis: a clinical-experimental investigation in normal and anomalous innervations. Acta Orthop Scand. 1966;S87:1-176.

44. Jeanne M. La deformation du pouce dans la paralysie cubitale. Mul Mém Soc Chir Paris. 1915;41:703-19.

45. Tsujino A, Macnicol MF. Finger flexion sign for ulnar neuropathy. J Hand Surg [Br]. 1998;23(2):240-1.

46. Earle AS, Vlastou C. Crossed fingers and other tests of ulnar nerve motor function. J Hand Surg [Am]. 1980;5(6):560-5.

47. Forrester-Brown M. Difficulties in the diagnosis of nerve function. J Surg [Br]. 1920;7:495-501.

48. Pollock L. Supplementary muscle movements in peripheral nerve lesions. Archs Neurol Psychiat. 1919;2:518-31.

49. Sunderland S. Voluntary movements and the deceptive action of muscles in peripheral nerve lesions. Aust N Z J Surg. 1944; 13:160-83.

50. Egawa T. Electromyographic studies on finger motion. J Osaka Univ Med School. 1959;11:1739-58.

51. Aiache A, Delagi EF. A pure sign of lumbrical function. Plast Reconstr Surg. 1974;53:312-5.

52. Sunderland S. The actions of the extensor digitorium communis, interosseous and lumbrical muscles. Am J Anat. 1945;77: 189-217.

53. Wartenberg R. A sign of ulnar palsy. JAMA. 1939;112:1688.

54. Sunderland $S$. The significance of hypothenar elevation in movements of opposition of the thumb. Aust NZJ Surg. 1944;13:155-6.

55. Mumenthaler M. Die ulnarisparesen. Stuttgart, Germany: Georg Thieme Verlag, 1961.

56. Pleet AB, Massey EW. Palmaris brevis sign in neuropathy of the deep palmar branch of the ulnar nerve. Ann Neurol. 1978;3:468-9.

57. Bowden R, Napier JR. The assessment of hand function after peripheral nerve injuries. J Bone Joint Surg [Br]. 1961;43:481-92.

58. Kapandji A. La défaillance du crochet ulnaire: ou encore "signe de la lime à ongles," signe peu connu d'atteinte du nerf ulnaire. Ann de Chir de la Main. 1999;18:295-8. 


\section{JHT Read for Credit \\ Quiz: Article \# 130}

Record your answers on the Return Answer Form found on the tear-out coupon at the back of this issue. There is only one best answer for each question.

\#1. Ulnar nerve involvement may have motor and sensory signs. This study reports on
a. motor signs
b. sensory signs
c. both motor and sensory signs
d. CNS signs

\#2. The design of the study is best described as
a. a narrative review
b. an RCT
c. a case report
d. a systematic review

\#3. Spurling's Test is best used to evaluate
a. cubital tunnel syndrome
b. cervical spine impingement
c. plexopathies of the superior brachial plexus

d. plexopathies of the inferior brachial plexus \#4. Jeanne's sign is
a. a mixed motor and sensory sign
b. a sensory sign
c. a motor sign
d. an electrodiagnostic finding

\#5. Comparing all the tests described in this study one can reasonably conclude that

a. there is a high degree of sensitivity and specificity throughout

b. there is a high degree of variability in the sensitivity and specificity throughout

c. none of the tests had a high degree of sensitivity

d. none of the tests had a high degree of specificity

When submitting to the HTCC for re-certification, please batch your JHT RFC certificates in groups of 3 or more to get full credit. 